\title{
How Evolutionary Theory Illuminates Radiologic Practice: Bipedalism
}

\author{
Richard B. Gunderman MD, PhD
}

Abdul R. S. Aasar BS

Indiana University School of Medicine, 702 North Barnhill Drive, Room 1053, Indianapolis, IN 46202

Key Words: Bipedalism; upright posture; radiology; radiologists

Upright we are, and we experience ourselves in this specific relation to the world. Erwin Straus, "The Upright Posture"

Many of the medical complaints that prompt routine radiologic imaging can be traced to the fact that human beings are not perfectly adapted to one of the most distinctive features of our species, upright posture. We walk upright on our hind limbs - a feature not found in any other nonhuman mammal in evolutionary history. ${ }^{1}$ Evolutionary biologists have been investigating this property for decades, but many of their more intriguing findings remain largely unknown to radiologists.

Bipedalism raises questions for radiologists. How did it evolve? What advantages does it afford, and what anatomic and physiologic challenges does it give rise to, particularly in the spine, pelvis, and lower limbs $?^{2}$ Gaining a deeper understanding of these questions can help radiologists better understand the genesis of a diverse range of conditions we encounter on a daily basis, such as traumatic and insufficiency fractures, osteoarthritis, spondylolisthesis, and even disorders with no apparent relationship to upright posture.

\section{Bipedalism}

An inspection of the fossil record suggests that bipedalism probably appeared in human ancestors approximately 7 million years ago. ${ }^{3}$ The musculoskeletal modifications that have permitted upright

This is the author's manuscript of the article published in final edited form as:

Gunderman, R. B., \& Aasar, A. R. S. (2018). How Evolutionary Theory Illuminates Radiologic Practice: Bipedalism. Academic Radiology, 25(12), 1667-1668. https://doi.org/10.1016/j.acra.2018.08.005 
posture involve the entire skeleton from head to toe, and make such adaptations easy to identify in the paleontological record. For example, the species Sahelanthropus, thought to have appeared at about the same time that the chimpanzee and human branches started to diverge, exhibits a relatively anteriorly placed foramen magnum, a requirement for the transition to an upright spine. ${ }^{3}$

More certain is that by 4 million years ago, species such as Australopithecus afarensis had emerged. ${ }^{4}$ They appear to have lived in open areas near dense woods, where upright posture increased their height and visual range, enabling them to recognize predators from a greater distance. Their forelimbs, now freed of locomotion, could be used for carrying, climbing, and manipulating objects. By about 2.5 million years ago, human ancestors had evolved a lumbar lordosis and thoracic kyphosis, and by 1.9 million years ago, greater hip support had appeared, permitting full bipedalism.5, 6

Interestingly, bipedalism appears to have predated the development of the expanded human brain by a substantial period of time. ${ }^{1}$ This suggests that the development of a larger brain was not the cause but the effect of freeing the forelimbs for more complex activities such as making and using tools. Instead of developing the excess capacity of a larger brain and then finding uses for it, it appears that human progenitors began to place increased demands on their brains, which grew over time to meet the challenge.

Of course, bipedalism also gave rise to a new group of challenges. Upright posture required a host of radical changes to the human skeleton and the muscles that support it. Ambulation requires an animal to create adequate friction between itself and the ground while producing adequate substrate reaction forces necessary for propulsion. During this process, mechanisms must be in place to protect the animal's musculoskeletal anatomy from potentially harmful mechanical loads, particularly on the cartilaginous articular surfaces of joints. $^{2}$

\section{Incomplete Adaptations}


Consider, for example, the sigmoidal curve of the human spine, which enhances the ability to balance the upper body over the hips and feet and serves as a shock absorber when we walk. The spine originally developed parallel to the ground, not perpendicular to it, and the increased load born by the bipedal spine predisposes to a host of spine-related problems. One is compression fractures, which are far more likely to occur in patients suffering from osteoporosis. Likewise, the increase in axial loading, particularly when extra weight is born by the upper limbs, increases the risk of spondylolysis.

Increased load bearing by the intervertebral discs increases the risk of disc ruptures, protrusions, and herniations. Various forms of misalignment, such as abnormal kyphosis, lordosis, and scoliosis, are also more likely. These problems are virtually exclusive to humans. Other mammals, including closely related mammals such as chimpanzees and gorillas, are less likely to suffer from these pathologies, because they operate bipedally only part of the time and are less likely to carry loads in their arms. ${ }^{2}$

With bipedalism, the hip joints, thighs, and knees all enlarged to better support the increased load on the hind limbs. But at about the time hominids became bipedal, the hips and knees began showing osteoarthritic changes. At the root is a tension between joint stability and efficiency of locomotion. The knee, the largest joint in the human body, is modeled on a hinge with three articulations: the patellofemoral, medial, and lateral tibiofemoral articulations, each of which is liable to osteoarthritic change, particularly with time and overuse. ${ }^{2}$

Upright posture has also affected the feet. What in hominids was once a grasping appendage used for climbing now functions primarily in support and propulsion. This dramatic shift in function requires radical alterations in structure that remain incomplete. For example, the human foot has evolved a pedal arch with a permanently anteriorly directed great toe. While allowing the foot to serve as both a propulsion lever and an energy dissipater, these imperfect adaptations also increase the risk of collapse of the plantar arch (pes planus), bunions, tendonitis, bursitis, and arthritides. ${ }^{2}$ 
Such deficiencies are exacerbated by the long human lifespan. Compared to other large-bodied hominids, human beings exhibit delayed maturation and survive decades longer. This provides time for the development of so-called degenerative diseases, such as degenerative arthritis in the spine, hips, knees, and feet. The fact that such conditions generally do not develop so early in life that they impair reproductive fitness suggests that there may be little evolutionary pressure to reduce them.

Of course, the costs of upright posture are not confined to the hind limbs. Wrist and elbow injuries due to falls would not occur if the forelimbs were in contact with the ground. Because they are elevated far above it, considerable potential energy is available to be transferred to such bones in a fall. In patients at increased risk for falls, the use of appliances such as walking sticks, walkers, and wheelchairs help to prevent such injuries.

As bipedalism stimulated brain growth, it also created challenges related to parturition. Gestation had to be shortened to permit passage of the large fetal head through the birth canal, meaning that humans are born relatively early in a state of prolonged helplessness and dependency. The large fetal cranium means that labor lasts considerably longer than in other animal species, potentially increasing both maternal and fetal mortality. The shape of pelvis, particularly in females, is adapted to these constraints. For example, the sacrum is shortened, to allow for a more capacious birth canal.

Another challenge with upright posture is balance. To keep an upright body with its high center of gravity erect, many adaptations are necessary, involving the visual system, the vestibular system, and many internal position sensors, which operate in concert to maintain posture and balance. Such challenges are only magnified during locomotion. Many processes can interfere with balance, including vision loss, inner ear disease, and declines in the central nervous system associated with aging — all increasing the risk of injury.

\section{Future Prospects}


In the future, further adaptation is likely to operate less by genetic than by behavioral, medicinal, and technological means. Maintaining a healthy weight can do much to reduce excess loading on bones and joints. ${ }^{7}$ Likewise, regular weight-bearing exercise can help to prevent osteoporosis and enhance the strength of supporting musculature. For example, building up the abdominal and paraspinal muscles increases both spinal stability and shock absorption, and the same goes for the hip and knee joints. Other possibilities include the use of exoskeletons and stem cell injections for arthritic joints.8, 9

Bipedalism offers many important benefits of which human beings are the happy beneficiaries, but it also imposes burdens that most human beings, particularly those who live long enough, are likely to confront at some point in life. While understanding these incomplete biological adaptations offers little as a means of enhancing diagnostic acumen, it does help to make sense of a large range of otherwise seemingly unrelated diseases and injuries that radiologists encounter on a daily basis. 


\section{References}

1. Lovejoy CO. Evolution of the human lumbopelvic region and its relation-ship to some clinical deficits of the spine and pelvis. In: Vleeming A, ed. Movement, Stability \& Lumbopelvic Pain 2nd ed, London: Churchill Liv-ingstone; 2007:141-158.

2. Latimer B. The perils of being bipedal. Ann Biomed Eng 2005; 33:3-6.

3. Brunet M, Guy F, Pilbeam D, et al. A new hominid from the upper Miocene of Chad, Central Africa. Nature 2002; 418:145-151.

4. Kimbel WH, Johanson DC, Rak Y. The first skull and other new discoveries of Australopithecus afarensis at Hadar, Ethiopia. Nature 1994; 368:449-451.

5. Dart RA. Australopithecus africanus, the man-ape of South Africa. Nature 1925; 115:195-199.

6. Simpson SW, Quade J, Levin NE, et al. A female Homo erectus pelvis from Gona, Ethiopia. Science 2008; 322:1089-1092.

7. Koonce R, Bravman J. Obesity and osteoarthritis: more than just wear and tear. J Am Acad Orthop Surg 2013; 21:161-169.

8. Zhang H. Design of a passive exoskeleton spine Masters Thesis. Design of a passive exoskeleton spine, 123; 2014. 2014.

9. Diekman BO, Guilak F. Stem cell-based therapies for osteoarthritis: challenges and opportunities. Curr Opin Rheumatol 2013; 25:119-126. 